\title{
Parametric Admissible Control for Descriptor Linear Systems via State-Derivative Feedback
}

\author{
Haihua $\mathrm{Yu}^{*}$ \\ School of Electronics Engineering, Heilongjiang University, Harbin, 150080, China \\ *Corresponding author
}

\begin{abstract}
Based on the general solution to the proposed generalized Sylvester matrix equation, very simple and complete parametric solutions to the admissible control problem for linear descriptor systems via derivative feedback are established in two cases. With several simple requirements on the design parameters of the solution, $\operatorname{rank}\left[\begin{array}{ll}E & B\end{array}\right]$ number arbitrary finite closed-loop poles with arbitrary algebraic and geometric multiplicities were assigned, and the closed-loop system was regular and impulse-free. The effectiveness of the proposed approach is demonstrated by a numerical example.
\end{abstract}

Keywords-admissible control; descriptor linear systems; eigenstructure assignment; regularity; impulse-freeness

\section{INTRODUCTION}

In the general field of control systems, an important part is the study of descriptor linear system, which has attracted many researcher's interesting in the last several decades. Within the obtained results of linear systems, control system design usually means to design a certain type of controller such that the closed-loop system meets certain design criteria, and sometimes the controller itself satisfies certain requirements. Different design problems are often defined by various design criteria, for example, for descriptor linear systems, stabilization, eigenstructure assignment (ESA), regularization, normalization and impulse elimination.

A descriptor linear system is clled admissible if it is regular, stable and impulse-free. It is well known that stability is an important property of a system, and the solution to a descriptor linear system exists and is unique if it is regular ${ }^{[1]}$. Moreover, if the initial condition of a system is not consistent or a control is not sufficiently smooth, in the response of a descriptor linear system, a impulse may arise. In most cases, we do not expect these impulse terms, since they may give rise to saturation of control or even break down the system. Therefore the research of certain feedback controllers to eliminating the impulsive behavior of a descriptor linear system is important. Unfortunately, these three problem usually are studied separately, such as [2]-[11] considered the regularization problem of linear descriptor systems by various feedback respectively, and [12]-[16] studied the impulse elimination problem for linear descriptor systems, while [17]-[33] focused on the eigenstructure assignment (ESA) for the first, second or higher-order linear systems, respectively.

A linear descriptor system usually be represented by the following state-space model

$$
E \dot{x}(t)=A x(t)+B u(t)
$$

where $x \in \mathbf{R}^{n}$ is the state vector and $u \in \mathbf{R}^{r}$ is the input vector, $E, A$ and $B$ are both coefficient matrices with appropriate dimensions. For descriptor linear system (1), the ESA problem also been studied in many results (see, [24]-[33]). In [25]-[27] the ESA problem via state-proportional(P) feedback are discussed. In the condition of R-controllable, rankE finite poles are assigned. In [28], the eigenstructure assignment problem via state-derivative(D) feedback for system (1) are discussed and a complete parametric approach was proposed. [29] and [30] proposed a parametric approach for ESA problem for system (1) via output feedback, [31] obtained a solution, in the condition of C-controllable, to the ESA problem via PD feedback and assigned $n$ finite closed-loop poles. Unfortunately, the closed-loop regularity and impulse-freeness of the systems were not discussed in all the above papers. In [32], ESA problem via $P$ feedback was studied, rankE arbitrary finite closed-loop poles are assigned and the closed-loop system is regular. However, that result did not consider the D feedback and did not studied the closed-loop impulse-freeness. [33] discussed the admissible control via PD feedback, and the proposed approach assigns $\operatorname{rank}\left[\begin{array}{ll}E & B\end{array}\right]$ arbitrary finite closedloop eigenvalues and guarantees the closed-loop regularity and impulse-freeness.

This work disposes of the admissible control problem of system (1) via D feedback. Without loss of the generality, we supposed that $B$ are full column rank and the system coefficients satisfy the following assumptions.

Assumption 1: $\operatorname{rank}\left[\begin{array}{ll}s E-A & s B\end{array}\right]=n$, for all $s \in \mathbf{C}$.

Assumption 2: $\operatorname{rank}\left[\begin{array}{ll}E & B\end{array}\right] \stackrel{\text { def. }}{=} n_{0}=n$ or $\operatorname{rank} A=n$.

This paper gives general parametric solutions to the admissible control problem based on a the solution of matrix equation in two different situations. In the first case, $n_{0}=n, n$ finite closed-loop poles were assigned. In the second case, $n_{0}<n$ and $\operatorname{rank} A=n, n_{0}$ finite closed-loop poles were assigned. On the other hand, in both the two situations, the closed-loop system is impulse-free and regular.

The paper is composed of five parts. In section 2, the admissible control problem is proposed. Section 3 gives the solution to the proposed matrix equation. Section 4 discusses 
the general solutions in two situations. An illustrative numerical example is given in Section 5 .

\section{PROBLEM FORMULATION}

For descriptor system (1), consider the following statederivative feedback controller

$$
u(t)=-K \dot{x}(t)+v(t),
$$

where $K \in \mathbf{R}^{r \times n}, v(t)$ is the reference input. When controller (2) is substituted into system (1), we obtain the closed-loop system as follows:

$$
(E+B K) \dot{x}(t)=A x(t)+B u(t)
$$

In this paper, we aim to find a feedback controller in the form of (2), such that the closed-loop system (3) has $n_{0}$ finite poles. Without loss of generality, we suppose the finite Jordan form of the closed-loop system as follows.

$$
J=\operatorname{diag}\left(J_{1}, J_{2}, \ldots, J_{q}\right) \in \mathbf{C}^{n_{0} \times n_{0}},
$$

where

$$
J_{i}=\left[\begin{array}{cccc}
s_{i} & 1 & & \\
& s_{i} & \ddots & \\
& & \ddots & 1 \\
& & & s_{i}
\end{array}\right] \in \mathbf{C}^{p_{i} \times p_{i}}, i=1,2, \ldots, q, \sum_{i=1}^{q} p_{i}=n_{0},
$$

the finite eigenvalue matrix $V \in \mathbf{C}^{n \times n_{0}}$ is non-singular, and they satisfy

$$
(E+B K) V J=A V,
$$

where $s_{i}, i=1,2, \ldots, q$, are self-conjugate complex numbers, which represent the finite poles of the closed-loop system.

Problem 1: Find the parametric forms of $K \in \mathbf{R}^{r \times n}$, and $V \in \mathbf{C}^{n \times n_{0}}$, to ensure the admissibility of the close-loop system (3), that is, the following three conditions are met simultaneously:

1. the close-loop Jordan form is as (4), that is, equation (5) satifies;

2. system (3) is regular, that is, the $\operatorname{det}(s(E+B K)-A) \neq 0$ for some complex number $s \in \mathrm{C}^{[34]}$;

3. system (3) is impulse-free.

Letting

$$
W=K V,
$$

then equation (5) can be rewritten as

$$
A V-E V J=B W J,
$$

where $A, E \in \mathbf{R}^{n \times n}, B \in \mathbf{R}^{n \times r} \quad$ and $\quad J \in \mathbf{C}^{n_{0} \times n_{0}}$ are determined matrices, $V \in \mathbf{C}^{n \times n_{0}}$ and $W \in \mathbf{C}^{r \times n_{0}}$ are to be defined.

It is clear that we can get the feedback gain matrix $K$ with the help of (6) if we obtained matrices $V$ and $W$, Therefore, to find the solution of Problem 1, we first need to study the generalized Sylvester matrix equation (7).

\section{The SOlution to EQUATION (7)}

Reference [35] studied the solution to equation

$$
A V-E V J=B_{D} W_{D} J+B_{P} W_{P}
$$

In this part, we apply these results to equation (7). Denoting

$$
\begin{aligned}
V & =\left[\begin{array}{llll}
V_{1} & V_{2} & \cdots & V_{q}
\end{array}\right], W=\left[\begin{array}{llll}
W_{1} & W_{2} & \cdots & W_{q}
\end{array}\right], \\
V_{i} & =\left[\begin{array}{llll}
V_{i 1} & v_{i 2} & \cdots & v_{i p_{i}}
\end{array}\right], W_{i}=\left[\begin{array}{llll}
w_{i 1} & w_{i 2} & \cdots & w_{i p_{i}}
\end{array}\right], i=1,2, \ldots, q .
\end{aligned}
$$

Under the Assumption 1, we can find two unimodular matrices $P(s) \in \mathbf{R}^{n \times n}[s]$, and $Q(s) \in \mathbf{R}^{(n+r) \times(n+r)}[s]$, satisying

$$
P(s)[s E-A \quad s B] Q(s)=\left[\begin{array}{ll}
0 & I_{n}
\end{array}\right] .
$$

Then we get the following conclusion for the parametric solution to equation (7).

Lemma 1: Under the Assumption 1, the general solutions to the equation (7) are given by

$$
\begin{aligned}
& {\left[\begin{array}{c}
v_{i j} \\
w_{i j}
\end{array}\right]=Q\left(s_{i}\right)\left[\begin{array}{c}
f_{i j} \\
-P\left(s_{i}\right)\left(E v_{i(j-1)}+B w_{i(j-1)}\right)
\end{array}\right],} \\
& v_{i 0}=0, w_{i 0}=0, j=1,2, \ldots, p_{i}, i=1,2, \ldots, q,
\end{aligned}
$$

where $P(s), \quad Q(s)$ satisfy (8), and $f_{i j} \in \mathbf{C}^{r}, \quad j=1,2, \ldots, p_{i}$, $i=1,2, \ldots, q$, is a set of arbitrary vectors.

\section{Letting}

$$
Q(s)=\left[\begin{array}{cc}
N(s) & * \\
D(s) & *
\end{array}\right], N(s) \in \mathbf{R}^{n \times r}[s], D(s) \in \mathbf{R}^{r \times r}[s],
$$

which satisfies obviously 


$$
\operatorname{rank}\left[\begin{array}{c}
N(s) \\
D(s)
\end{array}\right]=r
$$

It is obvious that $N(s), D(s)$ satisfy

$$
(s E-A) N(s)+s B D(s)=0,
$$

which is called a left-coprime factorization.

Another result about the solution to matrix equation (7) can be stated as follows.

Lemma 2: Under the Assumption 1, the parametric solutions to the equation (7) are given by

$$
\left[\begin{array}{c}
v_{i j} \\
w_{i j}
\end{array}\right]=\sum_{k=0}^{j-1} \frac{1}{k !}\left[\begin{array}{c}
N^{(k)}\left(s_{i}\right) \\
D^{(k)}\left(s_{i}\right)
\end{array}\right] f_{i(j-k)}, j=1,2, \ldots, p_{i}, i=1,2, \ldots, q,
$$

where matrix polynomials $N(s)$ and $D(s)$ satisfy (10), and $f_{i j} \in \mathbf{C}^{r}, \quad j=1,2, \ldots, p_{i}, \quad i=1,2, \ldots, q$, is a set of arbitrary vectors.

\section{SOlution TO PROBlem 1}

With the help of (9) or (11), we get the parametric forms of matrices $V \in \mathbf{C}^{n \times n_{0}}$ and $W \in \mathbf{C}^{r \times n_{0}}$. In order that $K$ solved from (6) is a real matrix, we need the following requirement to the free parameters $\left\{f_{i j}\right\}$.

Constraint 1: $f_{i j}=\bar{f}_{l j}, \quad j=1,2, \ldots, p_{i}\left(p_{i}=p_{l}\right), \quad i, l=1,2, \ldots, q$, if $s_{i}=\overline{s_{l}}$.

In the following, we will study the solution to Problem 1 in two different situations.

\section{A. Case of $n_{0}=n$}

In this case, $V$ is the closed-loop eigenvector matrix. So it must satisfies the following basic requirement :

Constraint 2: $\operatorname{det} V \neq 0$.

Considering of the singularity of matrix $V$, we can easily get the solution to the gain matrix $K$ as follows:

$$
K=W V^{-1},
$$

which obviously is a complete parametric solution. According to (5), we have

$$
\begin{aligned}
(s(E+B K)-A) V & =s(E+B K) V-(E+B K) V J \\
& =(E+B K) V(s I-J) .
\end{aligned}
$$

Therefore we get, in view of Constrain 2

$$
\operatorname{det}(s(E+B K)-A)=\operatorname{det}(E+B K) \operatorname{det}(s I-J) .
$$

On one side, since we can choose $J$, such that, $\operatorname{det}(s I-J) \neq 0$, therefore, if $\operatorname{det}(E+B K) \neq 0$, the closed-loop (3) is regular. On the other side, $(E+B K) V=E V+B W$, in view of (6), therefore, the following constraint is a necessary condition of the closedloop regularity.

Constraint 3: $\operatorname{det}(E V+B W) \neq 0$.

Remark 1: Since $\operatorname{rank}\left[\begin{array}{ll}E & B\end{array}\right]=n$, there always exists parameter $\left\{f_{i j}\right\}$ to satisfy constraint 3 .

Furthermore, under Constraint 3, system (3) is a conventional linear system and impulse-free.

According to above deduction, in the case of $n_{0}=n$, we obtain the following conclusion.

Theorem 1: Suppose the descriptor linear system (1) satisfy Assumption 1 and $n_{0}=n$, and given the Jordan form as (4).

1 If there exists a set of parameters $f_{i j} \in \mathbf{C}^{r}$, $j=1,2, \ldots, p_{i}, \quad i=1,2, \ldots, q$, such that Constrains 1-3 are met, then Problem 1 has a solution.

2 When the above Constrains are satisfied, the solution to Problem 1 is given by (12).

B. Case of $n_{0}<n, \quad \operatorname{rank} A=n$

In this case, we view $V$ as the finite closed-loop eigenvector matrix. Defining the infinite closed-loop eigenvector matrix as $V_{\infty} \in \mathbf{R}^{n \times\left(n-n_{0}\right)}$, then following basic requirement must be satisfied.

Constraint 2': $\operatorname{det}\left[\begin{array}{ll}V & V_{\infty}\end{array}\right] \neq 0$.

To get the matrix $K$, we define an auxiliary matrix $W_{\infty} \in \mathbf{R}^{r \times\left(n-n_{0}\right)}$ satisfying

$$
W_{\infty}=K V_{\infty} .
$$

We have, combining (6) and (13),

$$
\left[\begin{array}{ll}
W & W_{\infty}
\end{array}\right]=K\left[\begin{array}{ll}
V & V_{\infty}
\end{array}\right]
$$

Then we get the following parametric solutions to the gain matrix $K$, in view of Constraint 2',

$$
K=\left[\begin{array}{ll}
W & W_{\infty}
\end{array}\right]\left[\begin{array}{ll}
V & V_{\infty}
\end{array}\right]^{-1} .
$$

In this case, we view $W_{\infty} \in \mathbf{R}^{r \times\left(n-n_{0}\right)}$ as a free parameter matrix existed in the solution to equation (6), which represents the extra freedom degree in the design problem other than the parameters $\left\{f_{i j}\right\}$. 

get $^{[34]}$

Defining $J_{\infty}$ the infinite closed-loop Jordan matrix, then we

$$
(E+B K) V_{\infty}=A V_{\infty} J_{\infty} .
$$

Combining the above equation with (5), yields

$$
A\left[\begin{array}{ll}
V & V_{\infty} J_{\infty}
\end{array}\right]=(E+B K)\left[\begin{array}{ll}
V J & V_{\infty}
\end{array}\right]
$$

Rewritten the above equation as

$$
A\left[\begin{array}{ll}
V & V_{\infty}
\end{array}\right] \operatorname{diag}\left(I_{n_{0}}, J_{\infty}\right)=(E+B K)\left[\begin{array}{ll}
V & V_{\infty}
\end{array}\right] \operatorname{diag}\left(J, I_{n-n_{0}}\right) .
$$

Furthermore, post-multiplying the above by $\operatorname{diag}\left(J^{-1}, I_{n-n_{0}}\right)$, we have

$$
A\left[\begin{array}{ll}
V & V_{\infty}
\end{array}\right] \operatorname{diag}\left(J^{-1}, J_{\infty}\right)=(E+B K)\left[\begin{array}{ll}
V & V_{\infty}
\end{array}\right],
$$

Thus, we obtain

$$
\begin{aligned}
(s(E+B K)-A)\left[\begin{array}{ll}
V & V_{\infty}
\end{array}\right] & =s A\left[\begin{array}{ll}
V & V_{\infty}
\end{array}\right] \operatorname{diag}\left(J^{-1}, J_{\infty}\right)-A\left[\begin{array}{ll}
V & V_{\infty}
\end{array}\right] \\
& =A\left[\begin{array}{ll}
V & V_{\infty}
\end{array}\right]\left(s \operatorname{siag}\left(J^{-1}, J_{\infty}\right)-I\right) .
\end{aligned}
$$

When Constrain 2' is satisfied, we have

$$
\operatorname{det}(s(E+B K)-A)=\operatorname{det} A \operatorname{det}\left(\operatorname{sdiag}\left(J^{-1}, J_{\infty}\right)-I\right) .
$$

Since we can choose $J$ and $J_{\infty}$, such that, $\operatorname{det}\left(\operatorname{sdiag}\left(J^{-1}, J_{\infty}\right)-I\right) \neq 0$. Therefore, under the assumption $\operatorname{det} A \neq 0$, the closed-loop system is regular.

Considering the structure of matrix $J$ and $J_{\infty}$, we have, if $s_{i}<0$,

$$
\operatorname{det}\left(\operatorname{sdiag}\left(J^{-1}, J_{\infty}\right)-I\right)=(-1)^{n-n_{0}} \prod_{i=1}^{q}\left(s_{i}^{-1} s-1\right)^{p_{i}},
$$

thus,

$$
\operatorname{deg} \operatorname{det}(s(E+B K)-A)=\sum_{i=1}^{q} p_{i}=n_{0}
$$

On one hand, the closed-loop system is impulse-free if $\operatorname{rank}(E+B K)=n_{0}$ (see [34]). On the other hand, we have, in view of (6),

$$
(E+B K)\left[\begin{array}{ll}
V & V_{\infty}
\end{array}\right]=\left[E V+B W \quad E V_{\infty}+B W_{\infty}\right]
$$

Therefore, the following constrain is a necessary condition for the closed-loop impulse-freeness.

Constraint 3': $\operatorname{rank}\left[E V+B W \quad E V_{\infty}+B W_{\infty}\right]=n_{0}$.

Remark 2: If $\operatorname{rank}\left[\begin{array}{ll}E & B\end{array}\right]=n_{0}$, there exist parameter $\left\{f_{i j}\right\}$, $V_{\infty}$ and $W_{\infty}$, such that the above constraint is met.

According to the above deduction, in the case of $n_{0}<n, \operatorname{rank} A=n$, we obtain the following theorem.

Theorem 2: Suppose the descriptor linear system (1) satisfy Assumption 1 and $n_{0}<n, \operatorname{rank} A=n$.

1 If there exist a set of parameters $f_{i j} \in \mathbf{C}^{r}, j=1,2, \ldots, p_{i}$, $i=1,2, \ldots, q$, and matrices $V_{\infty} \in \mathbf{R}^{n \times\left(n-n_{0}\right)}, \quad W_{\infty} \in \mathbf{R}^{r \times\left(n-n_{0}\right)}$, such that Constrains 1, 2', and $3^{\prime}$ are satisfied, then Problem 1 has a solution.

2 When the above Constrains are satisfied, the solution to Problem 1 can be obtained by (14).

\section{NUMERICAL EXAMPLE}

Consider a system in the form of (1) with the follow matrix coefficients:

$$
E=\left[\begin{array}{llll}
0 & 1 & 0 & 0 \\
0 & 0 & 0 & 1 \\
0 & 0 & 0 & 0 \\
0 & 0 & 0 & 0
\end{array}\right], A=\left[\begin{array}{llll}
1 & 0 & 0 & 0 \\
0 & 1 & 2 & 0 \\
0 & 0 & 1 & 1 \\
0 & 1 & 1 & 0
\end{array}\right], B=\left[\begin{array}{ll}
0 & 0 \\
1 & 0 \\
0 & 1 \\
0 & 0
\end{array}\right] .
$$

For this system, we have $n=4, r=2, n_{0}=\operatorname{rank}\left[\begin{array}{ll}E & B\end{array}\right]=3<n$. In this example, we define the following finite Jordan matrix:

$$
J=\operatorname{diag}(-2,-3,-4)
$$

The two polynomials $N(s)$ and $D(s)$ satisfying (10) can be obtained as:

$$
N(s)=\left[\begin{array}{cc}
-s^{2} & 0 \\
-s & 0 \\
s & 0 \\
-s & s
\end{array}\right], D(s)=\left[\begin{array}{cc}
s+1 & -s \\
0 & 1
\end{array}\right]
$$

According to Corollary 2, we have the parametric solution to $V, W$ as

$$
v_{i}=\left[\begin{array}{c}
-s_{i}^{2} f_{i 1} \\
-s f_{i 1} \\
s f_{i 1} \\
-s f_{i 1}+f_{i 2}
\end{array}\right], w_{i}=\left[\begin{array}{c}
\left(s_{i}+1\right) f_{i 1}-s_{i} f_{i 2} \\
f_{i 2}
\end{array}\right], i=1,2,3,
$$


with $f_{i}=\left[\begin{array}{ll}f_{i 1} & f_{i 2}\end{array}\right]^{\top}, \quad i=1,2,3$.

Simply choosing the parameters $f_{i}, i=1,2,3$, as:

$$
f_{1}=\left[\begin{array}{l}
1 \\
0
\end{array}\right], f_{2}=\left[\begin{array}{l}
0 \\
1
\end{array}\right], f_{3}=\left[\begin{array}{l}
1 \\
1
\end{array}\right],
$$

we can easily compute

$$
V=\left[\begin{array}{ccc}
-4 & 0 & 16 \\
2 & 0 & 4 \\
-2 & 0 & -4 \\
2 & -3 & 0
\end{array}\right], W=\left[\begin{array}{ccc}
-1 & 3 & 1 \\
0 & 1 & 1
\end{array}\right]
$$

Further taking

$$
V_{\infty}=\left[\begin{array}{l}
0 \\
1 \\
0 \\
0
\end{array}\right], W_{\infty}=\left[\begin{array}{l}
1 \\
1
\end{array}\right],
$$

which satisfy Constraints 1, 2' and 3', we obtain the control feedback gains as

$$
K=\left[\begin{array}{llll}
0.1250 & 1.0000 & 0.2500 & -1.0000 \\
0.0417 & 1.0000 & 0.5833 & -0.3333
\end{array}\right]
$$

It can be verified that

$$
\operatorname{det}\left(s E_{c}-A\right)=s^{3} / 24+3 s^{2} / 3+13 s / 12+1,
$$

and

$$
\operatorname{rank}(E+B K)=3 .
$$

which means

$$
\operatorname{deg} \operatorname{det}\left(s E_{c}-A_{c}\right)=\operatorname{rank} E_{c}=n_{0}=3 .
$$

There the closed-loop system is impulse-free, stable and regular.

\section{ACKNOWLEDGMENT}

This work is partially supported by Key Laboratory of Electronics Engineering, College of Heilongjiang Province, (Heilongjiang University), P. R. China (No. DZGC201611) and Science and Technology Innovative Research Team in Higher Educational Institutions of Heilongjiang Province (No. 2012TD007).

\section{REFERENCES}

[1] E. L. Yip, and R. R. Sincovec, "Solvability, controllability, and observability of continous descriptor systems," IEEE Transaction on Automatic Control, vol. 26, no. 3, pp 702-707, 1981

[2] A. Bunse-Gerstner, V. Mehrmann, and N. K. Nichols, "Regularization of descriptor systems by derivative and proportional state feedback," SIAM Journal of Matrix Anylasis and Applications, vol. 13, no. 1, pp. 46-67, 1992.

[3] L. R. Fletcher, "Regularizability of descriptor systems," International Journal of Systems Science, vol. 17, no. 6, pp. 843-847, 1986.

[4] D. L. Chu, and D. W. C. Ho, "Necessary and sufficient conditions for the output feedback regularization of descriptor systems," IEEE Transactions on Automatic Control, vol. 44, no. 2, pp. 405-412, 1999.

[5] D. L. Chu and D. W. C. Ho, "Regularization of singular systems by derivative and proportional output feedback," SIAM Journal of Matrix Anylasis and Applications, vol. 19, no. 1, pp. 21-38, 1998.

[6] V. Lovass-nagy, D. L. Powers, and R. J. Schilling, “On regularizing descriptor systems by output feedback," IEEE Transaction on Automatic Control, vol. 39, no.7, pp. 1507-1509, 1994.

[7] D. H. Wang, and C. B. Soh, "On regularizing singular systems by decentralized output feedback,” IEEE Transaction on Automatic Control, vol. 44, no. 1, pp. 148-152, 1999.

[8] R. Byers, P. Kunkel, and V. Mehrmann, "Regularization of linear descriptor systems with variable coefficients," Siam Journal on Control and Optimization, vol. 35, no. 1, pp. 117-133, 1997.

[9] G. R. Duan and X. Zhang, "Regularization of linear descriptor systems via output plus partial state derivative feedback," Asian Journal of Control, vol. 5, no. 3, pp. 334-340, 2003.

[10] W. J. Mao and J. Chu, "Regularisation and stabilisation of linear discrete-time descriptor systems,” IET Control Theory and Applications, vol. 4, no. 10, pp. 2205-2211, 2010.

[11] T. Berger and T. Reis, "Regularization of linear time-invariant differential-algebraic systems," Systems and Control Letters, vol. 78, no. 4, pp. 40-46, 2015

[12] G. R. Duan and A. G. Wu, "Impulse elimination via P-D feedback in descriptor linear systems," Dynamics of Continuous, Discrete and Impulsive System, Series A - Mathematical Analysis, no. 3, pp. 714-721, 2005.

[13] G. R. Duan. and A. G. Wu, "Impulse elimination via state feedback in descriptor linear systems, Dynamics of Continuous," Discrete and Impulsive System, Series A - Mathematical Analysis, no. 3, pp. 722-729, 2005.

[14] A. G. Wu, G. R. Duan and S. M. Zhao, "Impulsive-mode controllablisability in descriptor linear s ystems," IET Control Theory and Applications, vol. 1, no. 3, pp. 558-563, 2007.

[15] A. G. Wu, G. R. Duan, and H. H. Yu, "Impulsive-mode controllablizability revisited for descriptor linear systems," Asian Journal of Control, vol. 11, no. 3, pp. 358-365, 2009.

[16] J. Li, Y. F. Teng, Q. L. Zhang, J. H. Li and L. Qiao, "Eliminating impulse for descriptor system by derivative output feedback,” Journal of Applied Mathematics, 265601, 2014.

[17] A. Bhaya and C. Desoer, "On the design of large flexible space structues (LFSS),” IEEE Transactions on Automatic Control, vol. 30, no. 11, pp. 1118-1120, 1985.

[18] J. N. Juang, K. B. Lim and J. L. Junkins, "Robust eigensystem assignment for flexible structures," Journal of Guidance, Control and Dynamics, vol. 12, no. 3, pp. 381-387, 1989.

[19] E. K. Chu and B. N. Datta, "Numerically robust pole assignment for second-order systems,” International Journal of Control, vol. 64, no. 4, pp. 1113-1127, 1996

[20] Y. Kim and H. S. Kim, "Eigenstructure assignment algorithm for mechanical second-order systems,” Journal of Guidance, Control and Dynamics, vol. 22, no. 5, pp. 729-731, 1999.

[21] G. R. Duan and H. H. Yu, "Robust pole assignment in high-order descriptor linear systems via proportional plus derivative state feedback.” IET Control Theory and Applications, vol. 2, no. 4, pp. $277-$ 287, 2008. 
[22] H. H. Yu and G. R. Duan, "ESA in high-order linear systems via output feedback,” Asian Journal of Control, vol. 11, no. 3, pp. 336-343, 2009.

[23] H. H. Yu and G. R. Duan, "ESA in high-order descriptor linear systems via output feedback," International Journal of Control Automation and Systems, vol. 8, no. 2, pp. 408-417, 2010.

[24] D. J. Cobb, "Feedback and pole placement in descriptor variable sytems," International Journal of Control, vol. 33, pp. 1135-1146, 1981.

[25] G. R. Duan, "Solution to matrix equation $A V+B W=E V F$ and eigenstructure assignment for descriptor systems," Automatica, vol. 28, no. 3, pp. 639-643, 1992.

[26] G. R. Duan, "Eigenstructure assignment and response analysis in descriptor linear systems with state feedback control.” International Journal of Control, vol. 69, no. 5, pp. 663-694, 1998.

[27] G. R. Duan, "Right coprime factorizations for single-input descriptor linear systems: A simple numerically stable algorithm.” Asian Journal of Control, vol. 4, no. 2, pp. 146-158, 2002.

[28] H. S. Abdelaziz, Taha, "Parametric eigenstructure assignment using state-derivative feedback for linear systems,” Journal of Vibration and Control, vol. 18, no. 12, pp. 1809-1827, 2012.

[29] G. R. Duan, "Parametric approach for eigenstructure assignment in descriptor systems via output feedback," IEE Proceedings: Control Theory and Applications, vol. 142, no. 6, pp. 611—616, 1995.

[30] G. R. Duan, J. Lam and G. P. Liu, "Robust eigenvalue assignment in descriptor systems via output feedback.” Asian Journal of Control, vol. 6, no. 1, pp. 145-155, 2004.

[31] G. R. Duan, and Patton R.J., Eigenstructure assignment in descriptor linear systems via propotional plus derivative state feedback, Internatioal Journal of Control, 1997, 68(5): 1147-1162.

[32] G. R. Duan and R. J. Patton, "Eigenstructure assignment in descriptor systems via state feedback-A new complete parametric approach," International Journal of Systems Science, vol. 29, no. 2, pp. 167-178, 1998.

[33] H. H. Yu, "Parametric stabilization for descriptor linear systems via state-proportional and -derivative feedback," Journal of the Franklin Institute-Engineering and Applied Mathematics vol. 353, no. 5, pp. 992-1004, 2016.

[34] G. R. Duan, Analysis and Design of Descriptor Linear Systems, Springer, 2010.

[35] H. H. Yu and D. G. Bi, "Solution to generalized matrix equation $A V-E V J=B_{D} W_{D} J+B_{P} W_{P}$, " Proceedings of 33th Chinese Control Conference, pp. 3857-3862, 2014. 